\title{
Das Patientenmagazin der DRG
}

Patientenmagazin Reloaded: mit ganz neuen Möglichkeiten für Sie als Praxisinhaber oder Leiter einer radiologischen Abteilung

- Sie leiten eine radiologische Praxis oder Abteilung und möchten das Patientenmagazin bestellen?

- Ihr Unternehmen möchte eine Anzeige in unserem Magazin schalten?

Dann rufen Sie uns an, oder schreiben Sie uns eine Mail:

patientenmagazin@drg.de

\section{Unser Partner:}

TORNER BRAND MEDIA GMBH

Ralph Burkhardt

Barmbeker Straße 5a

22303 Hamburg

Mail: patientenmagazin@drg.de

Fon: $040300327-55$

Fax: 040 300327-70

www.torner.cc

\section{Herausgeber:}

Deutsche Röntgengesellschaft e. V.

Ernst-Reuter-Platz 10

10587 Berlin

www.drg.de

\section{Interview}

Warum ein Radiologie-Magazin für Patienten, wie kam die DRG dazu? Zu den Hintergründen der Entstehung und Weiterentwicklung des Hefts - und wieso es für Leser und Praxen gleichermaßen attraktiv ist - lesen Sie im Interview mit DRG-Geschäftsführer Dr. Stefan Lohwasser:

\section{Patientenmagazin Reloaded}

Seit drei Jahren publiziert die Deutsche Röntgengesellschaft (DRG) das Radiologie-Magazin MEDIZIN MIT DURCHBLICK. Ab der neuesten Ausgabe vom November 2016 gibt es für die Bezieher des Hefts, also alle PraxisinhaberInnen oder KlinikleiterInnen völlig neue Möglichkeiten der Mitgestaltung. Dr. Stefan Lohwasser, Geschäftsführer der DRG hat die Vorteile im Interview zusammengefasst.

Herr Dr. Lohwasser, wieso gibt es ein Radiologie-Magazin für Patienten?

Lohwasser: Die Deutsche Röntgengesellschaft hat 2011 eine repräsentative Umfrage in Auftrag gegeben, die eines deutlich gezeigt hat: Die Möglichkeiten der modernen Radiologie in Diagnostik und Therapie sind entweder nicht bekannt oder werden anderen Fachgebieten zugeordnet!

\section{Das heißt, die meisten Leute wissen über- haupt nicht, was die Radiologie alles kann?}

Genau! Daraufhin hat Professor Michael Forsting, Leiter des Instituts für Diagnostische und Interventionelle Radiologie und Neuroradiologie am Uniklinikum Essen, die Initiative 'Patientenmagazin' gestartet - und nun gibt die DRG bereits seit 2013 MEDIZIN MIT DURCHBLICK heraus.

\section{Wie ist das Heft aufgebaut?}

Das publikumswirksame Format erzählt Geschichten aus der Radiologie, erklärt Wirkungsweisen bildgebender Diagnostik - und nimmt dabei den Patienten auch eventuelle Ängste vor einer radiologischen Untersuchung! Unser Radiologie-Magazin spielt damit eine wichtige Rolle bei der Information und Aufklärung von Patienten.

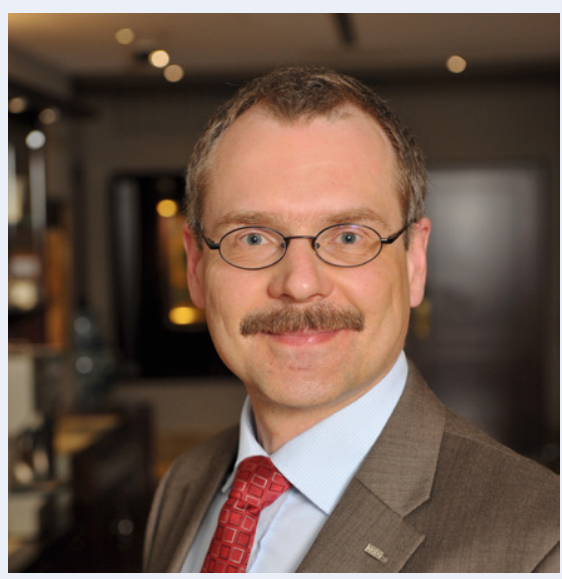

Dr. Stefan Lohwasser, DRG-Geschäftsführer

MEDIZIN MIT DURCHBLICK hat einen neuen Anstrich erhalten. Was ist neu?

Seit dem Relaunch im November 2016 ist die Erstellung von praxisindividuellen Ausgaben möglich, die ein wichtiger Baustein für die Bindung von Zuweisern und Patienten sein können. Wir sind gespannt auf die Rückmeldungen!

Vielen Dank für das Gespräch!

Blättern Sie durch unsere neueste Ausgabe von MEDIZIN MIT DURCHBLICK (Nov. 2016): http://www.torner-brand-media.de/DRG

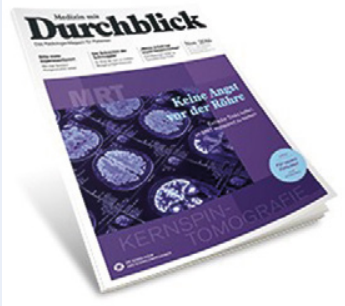

